\title{
Comparative Assessment of Performance of Aluminium Sulphate (Alum) and Ferrous Sulphate as Coagulants in Water Treatment
}

\section{Mbaeze $\mathrm{MC}^{1 *}$, Agbazue VE ${ }^{1}$ and Orjioke $\mathrm{NM}^{1,2}$}

${ }^{1}$ Department of Pure and Industial Chemistry, University of Nigeria, Nsukka, Nigeria

${ }^{2}$ Department of Science Laboratory Technology, University of Nigeria, Nsukka, Nigeria

"Corresponding author: Mbaeze MC, Department of Pure and Industial Chemistry, University of Nigeria, Nsukka, Nigeria, Tel: 234-803444735; E-mail: Chimambaeze@gmail.com

Rec date: September 28, 2017; Acc date: October 16, 2017; Pub date: October 23, 2017

Copyright: (C) 2017 Mbaeze MC, et al. This is an open-access article distributed under the terms of the Creative Commons Attribution License, which permits unrestricted use, distribution, and reproduction in any medium, provided the original author and source are credited.

\begin{abstract}
Alum and ferrous sulphate, being among the best-known coagulants in water treatment were investigated with the aim of determining their coagulation efficiencies. The parameter levels of a turbid water were determined before and after treatment with alum and ferrous sulphate respectively at coagulant dosages of 1 to $10 \mathrm{~g}$ per 3 litres of turbid water, for each of the following parameters: $\mathrm{pH}$, total suspended solids (TSS), dissolved oxygen (DO), biochemical oxygen demand (BOD5), turbidity, chloride, fluoride, phosphate and chemical oxygen demand (COD). The turbid water was prepared by addition of ground humus soil to tap water. Using a sedimentation beaker, experiments were conducted, leading to optimum coagulant dosage in some parameters. Coagulation experiments of the turbid water at the coagulant dosage of $10 \mathrm{~g}$ per 3 litres, gave the following coagulation efficiencies with alum as the coagulant: $\mathrm{pH}(44.92 \%)$, TSS (98.71\%), DO (90.10\%), BOD5 (100\%), Turbidity $(98.70 \%)$, chloride (100\%), fluoride (100\%), Phosphate $(80 \%)$, COD $(100 \%)$ and Copper $(0.00 \%)$. Similarly, using ferrous sulphate coagulant for the same level of turbid water and same dosage, the coagulation efficiencies achieved were: $\mathrm{pH}(57.24 \%)$, TSS (96.54\%), DO $(96.31 \%)$, BOD5 $(100 \%)$, Turbidity $(96.77 \%)$, and chloride $(100 \%)$, fluoride $(100 \%)$, phosphate (91.11\%), COD $(100 \%)$ and Copper $(0.00 \%)$. The results showed that increasing coagulant dosage enhances water quality with respect to all the parameters studied. Furthermore, the results indicated that $\mathrm{pH}, \mathrm{DO}, \mathrm{BOD} 5$, fluoride, phosphate and COD mean \% efficiencies were higher for ferrous sulphate coagulant in comparison with alum. On the other hand, alum displayed better coagulation efficiency than ferrous sulphate in the following parameters: TSS, turbidity and chloride. The overall results of the coagulation studies applying increasing coagulant dosage revealed that coagulant efficiency is parameter dependent. The outcome of this work can be an important guide to water treatment operators.
\end{abstract}

Keywords: Coagulation efficiency; Comparison; Water treatment; Alum; Iron (II) sulphate

\section{Introduction}

Water is a basic necessity of life. It is a limited resource. So, preserving the quality of water is important for the drinking water supply. Water quality can be compromised by the presence of harmful agents. One of the major problems of using surface water as source for drinking water is the high content of natural organic matter (NOM). NOM has an adverse effect on the aesthetic water quality and may increase corrosion and biofilm growth in the water distribution $[1,2]$. Furthermore, when water is disinfected, chemical disinfectants can react with NOM to form disinfection by-products (DBPs) like Trihalomethanes (THMs) and halo acetic Acids [3]. DBPs are considered to be potentially carcinogenic [3,4]. In 1998, the US Environmental Protection Agency (USEPA) regulated the THM and $\mathrm{HAA}$ at a maximum allowable level of 80 and $60 \mu \mathrm{g} / \mathrm{l}$ respectively for drinking water [5]. Hence, NOM has to be removed from drinking water more efficiently. In drinking water treatment, coagulation process is very important stage for the maintenance of acceptable treated water quality and economic plant operation [6]. Chemical coagulants are added to water to facilitate bonding among particulates that are widely used to enhance the removal of colloidal particles. Coagulation is not only effective in precipitation of particles, but also it has an important objective of removing pathogens. Many researchers have applied the coagulation to remove turbidity and to react with NOM [7].

Some of the well-known and common coagulants used are aluminium sulphate, ferric chloride and ferrous sulphate for water treatment7. Determining the optimum coagulant dosage for a given raw water is a major problem. Jar test procedure with a six-unit multiple stirrer system has been commonly used to determine the required concentration of coagulant dosage [7-11]. This is generally carried out periodically $[10,11]$. But a four litre sedimentation beaker with a stirring bar inside it, and mounted on magnetic stirrer is used for this research work to determine optimum coagulant dosage in some parameters.

Optimum coagulant dosage is the lowest coagulant dosage at which maximum coagulation efficiency is achieved. The following parameters were tested for coagulation efficiency: $\mathrm{pH}$, TSS, DO, BOD5, Turbidity, Chloride, Fluoride, Phosphate, COD and Copper. During the sedimentation beaker test the level of one factor is set differently while levels of other factors are held constant. By varying only one factor, the operator can see how changes to that factor will affect the treatment process results. The effectiveness of the coagulation process is highly dependent on optimum coagulant dosage, raw water $\mathrm{pH}$, mixing time and sedimentation time. Proper coagulation is essential to produce satisfactory treated water qualities, to maintain the economic value of 
the plant operation and also for DBP control. Poor control will cause wastage of chemicals, low water qualities and failure in the sedimentation and filtration processes.

In addition, excessive coagulant dosage has been linked to several medical disorders such as Alzheimer's disease [12]. When there is high turbidity in water the physical properties of water are affected. Due to the water quality problems and stricter regulations for drinking water quality, plant operators have to use the sedimentation beaker test to determine the required coagulant dosage at any time. Again, jar test has limitations in that it is expensive, and time-consuming [10-13]. Sedimentation beaker test is cheap, saves time and easy to operate. Furthermore, depending on the $\mathrm{pH}$ after the coagulant is added, two possible reactions are generally possible.

*With aluminium-based coagulants, the metal ion is hydrolysed to form aluminium hydroxide floc as well as hydrogen ions. The hydrogen ions will react with the alkalinity of the water and in the process, decrease the $\mathrm{pH}$ of the water as can be seen from the equation below: for alum.

$$
\begin{aligned}
& \mathrm{Al}_{2}\left(\mathrm{SO}_{4}\right)_{3 .} \cdot 16 \mathrm{H}_{2} \mathrm{O} \rightarrow 2 \mathrm{Al}^{3+}+3 \mathrm{SO}_{4}{ }^{2-}+16 \mathrm{H}_{2} \mathrm{O} \rightarrow 2 \mathrm{Al}(\mathrm{OH})_{3}+6 \mathrm{H}^{+}+3 \mathrm{SO}_{4}{ }^{2-} \\
& +10 \mathrm{H}_{2} \mathrm{O}-1
\end{aligned}
$$

Similarly, for ferrous sulphate, the following reaction takes place:

$$
\mathrm{FeSO}_{4} \cdot 7 \mathrm{H}_{2} \mathrm{O} \rightarrow \mathrm{Fe}^{2+}+\mathrm{SO}_{4}{ }^{2-}+7 \mathrm{H}_{2} \mathrm{O} \rightarrow \mathrm{Fe}(\mathrm{OH})_{2}+\mathrm{SO}_{4}{ }^{2-}+2 \mathrm{H}^{+}+5 \mathrm{H}_{2} \mathrm{O}-2
$$

The above hydrolysis reactions typically take place at a dosed water $\mathrm{pH}$ in the range 5.8 to 7.5 , depending on the particular coagulant. Colour and colloidal matter is removed by adsorption onto/within the metal hydroxide hydrolysis products that are formed, and is sometimes referred to as sweep-floc coagulation.

${ }^{\star}$ If an excess coagulant is added so that the dosed water $\mathrm{pH}$ is less than 5.0, then the metal ions will directly neutralize the negatively charged organic compounds and colloids in the raw water. This allows the organic molecules to contribute to floc formation and is often referred to as enhanced coagulation and is often done to boost the removal of disinfection by-product precursors [14].

Furthermore, industrial waters are clarified to remove turbidity and color from the effluent streams in the textile, paper and other polluting industries [15]. The dictionary meaning of a coagulant is an agent that induces curdling or congealing. In a water treatment, what it is, a chemical that will remove color and turbidity present in raw water in the form of flocs. Coagulants neutralize the repulsive electrical charges (typically negative) surrounding particles allowing them to "stick together" creating clumps or flocks. Flocculants facilitate the agglomeration or aggregation of the coagulated particles to form larger floccules and thereby hasten gravitational settling. Some coagulants serve a dual purpose of both coagulation and flocculation in that they create large floc's that readily settle.

In wastewater treatment, coagulation and flocculation are employed to separate suspended solids from water. Although the terms coagulation and flocculation are often used interchangeably, or the single term "flocculation" is used to describe both; they are in fact, two distinct processes. Knowing their differences can lead to a better understanding of the clarification and dewatering operations of wastewater treatment. Finely dispersed solids (colloids) suspended in wastewaters are stabilized by negative electric charges on their surfaces, causing them to repel each other. Since this prevents these charged particles from colliding to form larger masses, called flocs, they do not settle. To assist in removal of colloidal particles from suspension, chemical coagulation and flocculation are required. These processes, usually done in sequence, are a combination of physical and chemical procedures. Chemicals are mixed with wastewater to promote the aggregation of the suspended solids into particles large enough to settle or be removed.

Coagulation is the destabilization of colloids by neutralizing the forces that keep them apart. Cationic coagulants provide positive electric charges to reduce the negative charge (zeta potential) of the colloids. As a result, the particles collide to form larger particles (flocs). Coagulation thus implies formation of smaller compact aggregates. Rapid mixing is required to disperse the coagulant throughout the liquid. Care must be taken not to overdose the coagulants as this can cause a complete charge reversal and restabilize the colloid complex.

Effluents are heterogeneous in nature. Chemical coagulation is an important unit process in water treatment for the removal of turbidity. Its application in water treatment is followed by sedimentation and filtration. Various types of coagulants are being used to condition water before sedimentation and filtration [15].

\section{Materials and Methods}

\section{The apparatus required include:}

- $\mathrm{pH}-\mathrm{meter}$

- Magnetic stirrer

- Sedimentation beaker

- Stop watch

- 20 liters white gallon

- 1 liter gallon

- 5 liters gallon

- Conical flask $(250 \mathrm{ml})$

- $50 \mathrm{ml}$ beurette

- Heating mantle

- Whatman filter paper

- Electronic weighing balance

- Spectrophotometer

- Incubator

- Oven and minifurnance

\section{Materials/Reagents:}

- Buffer solutions of $\mathrm{pH} 6.8$ and distilled water

- Humus soil

- Conc. $\mathrm{H}_{2} \mathrm{SO}_{4}$

- Distilled water

- Clean tap water/Base water

- Aluminum sulphate $\mathrm{Al}_{2}\left(\mathrm{SO}_{4}\right)_{3} \cdot 18 \mathrm{H}_{2} \mathrm{O}$ Octadecahydrate (Alum)

- Ferrous sulphate crystalline: $\mathrm{FeSO}_{4} \cdot 7 \mathrm{H}_{2} \mathrm{O}$ Heptahydrate

Coagulant processing: The two coagulants used for this experiment which are Aluminum sulphate and iron (II) sulphate with chemical formulae $\mathrm{Al}_{2}\left(\mathrm{SO}_{4}\right)_{3} \cdot 18 \mathrm{H}_{2} \mathrm{O}$ and $\mathrm{FeSO}_{4} \cdot 7 \mathrm{H}_{2} \mathrm{O}$ respectively were purchased from scientific equipment stores at Ogige Main, Market Nsukka. The caked coagulants were crushed with ceramic pestle and mortar.

Soil sample processing: The humus soil which was collected from University of Nigeria, Nsukka Agricultural Farm was first destoned. It was ground into powder using ceramic pestle and mortar in the 
Citation: Mbaeze MC, Agbazue VE, Orjioke NM (2017) Comparative Assessment of Performance of Aluminium Sulphate (Alum) and Ferrous Sulphate as Coagulants in Water Treatment. Mod Chem Appl 5: 233. doi: 10.4172/2329-6798.1000233

Page 3 of 14

laboratory. The powder was sieved with a laboratory sieve of known mesh size to obtain a smooth texture of the material.

Collection of water samples: 20 litres tap water was collected from a running tap, using a clean 20 litre plastic container. The water was transferred to the laboratory for further studies and labeling of the samples.

Sample preparation: Preparation of synthetic turbid water for the coagulation experiment was done by adding ground humus soil prepared as described above to tap water. About 50-70 g of the material was added to 20 litres of tap water. The suspension was stirred with a stick for about 5-10 minutes to achieve a uniform dispersion of particles. Then it was allowed to settle for at least 5-10 minutes for complete hydration of the materials. A 5 litre volume of the synthetic turbid water was taken out and poured into a 5-litre gallon, tightly covered and shaken. The turbidity was determined and 1 litre of the sample preserved for the analysis of parameters. Three litres of the turbid water was transferred to a sedimentation beaker and a stirring bar put into it just before coagulation. A given weight of the coagulant was added and the stirrer switched on to stir the water with coagulant for 10 minutes for coagulation/flocculation. After 5 hours of sedimentation, 1 litre of clarified water was taken out for determination of the parameters. The experiment was performed using $1 \mathrm{~g}, 2 \mathrm{~g}, 3 \mathrm{~g}, 4 \mathrm{~g}, 5 \mathrm{~g}, 6 \mathrm{~g}, 7 \mathrm{~g}, 8 \mathrm{~g}, 9 \mathrm{~g}$, and $10 \mathrm{~g}$ respectively of each of the two coagulants (Alum and Ferrous Sulphate) at a time. The experimental set up as shown below in Figure 1.

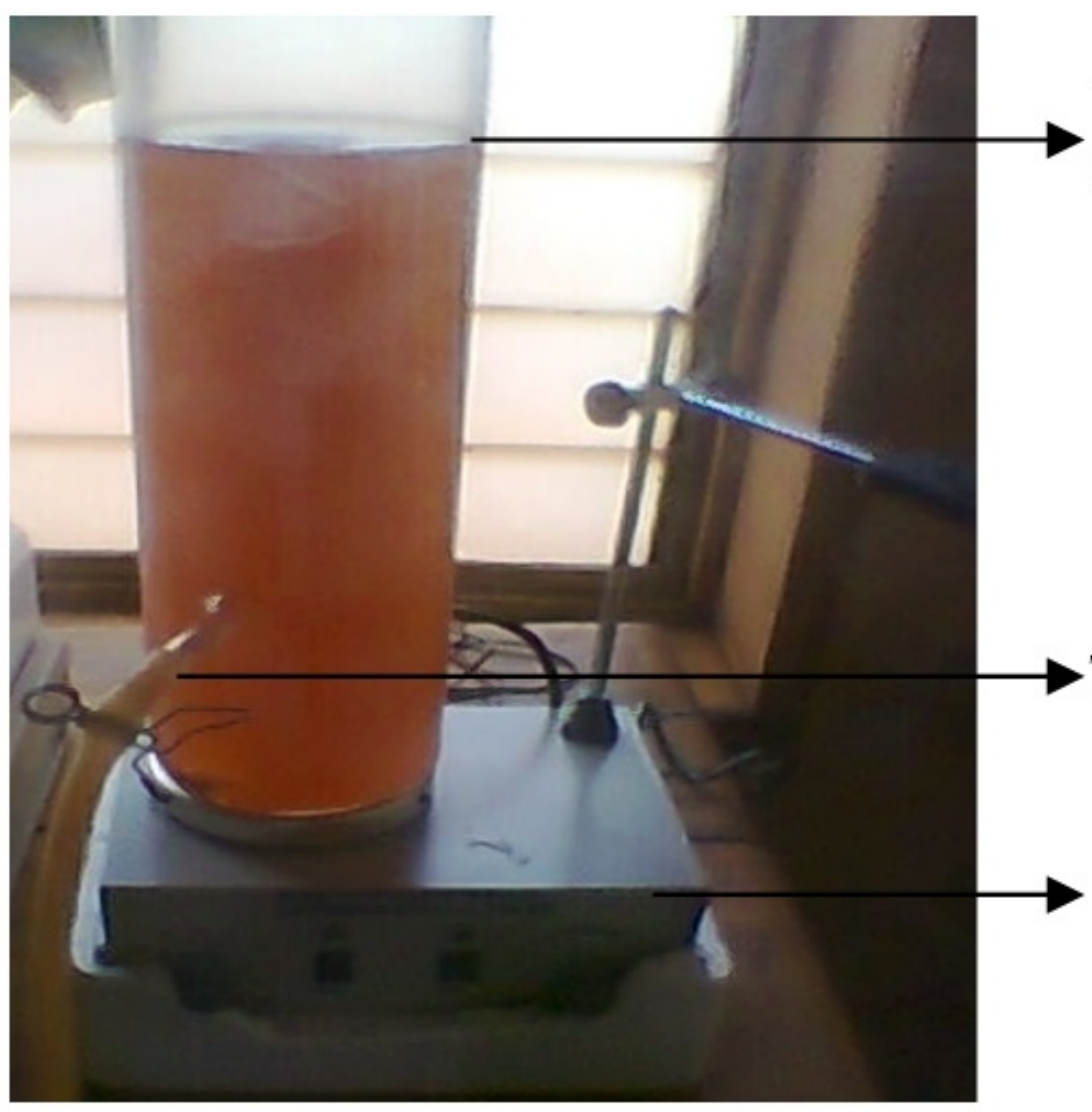

Turbid Water Level

Figure 1: A Sedimentation Beaker mounted on a magnetic stirrer (for coagulation/flocculation).

Standard methods adopted by American water works association/ water pollution control federation (AWWA, WPCF) and Association of Official Analytic Chemist (AOAC) are used for determination of the parameters listed above, before and after the coagulation experiment for each of the dosages stipulated. The same process was carried out for each of the two coagulants under study.

\section{Results and Discussion}

\section{Results}

The results of various levels of efficiency achieved by different doses of each of the coagulants are shown in Tables 1-10. Furthermore, the comparative variations in coagulant performance efficiency have been represented graphically in Figures 2-10. 
Citation: Mbaeze MC, Agbazue VE, Orjioke NM (2017) Comparative Assessment of Performance of Aluminium Sulphate (Alum) and Ferrous Sulphate as Coagulants in Water Treatment. Mod Chem Appl 5: 233. doi: 10.4172/2329-6798.1000233

Page 4 of 14

\begin{tabular}{|c|c|c|c|c|c|c|}
\hline \multirow[b]{2}{*}{ Parameter } & \multirow{2}{*}{$\begin{array}{l}\text { Dosage } \\
\text { (g) }\end{array}$} & \multirow[b]{2}{*}{$\mathbf{N}$} & \multicolumn{2}{|l|}{$\mathrm{Al}_{2}\left(\mathrm{SO}_{4}\right)_{3} \cdot 18 \mathrm{H}_{2} \mathrm{O}$} & \multicolumn{2}{|l|}{$\mathrm{FeSO}_{4} \cdot 7 \mathrm{H}_{2} \mathrm{O}$} \\
\hline & & & Mean \% efficiency & Std. Deviation & Mean \% efficiency & Std. Deviation \\
\hline & $1 \mathrm{~g}$ & 2 & 11.111 & 0 & 14.5833 & 0.98209 \\
\hline & $2 \mathrm{~g}$ & 2 & 15.3756 & 1.82586 & 16.0798 & 0.82994 \\
\hline & $3 \mathrm{~g}$ & 2 & 20.27 & 0 & 22.973 & 0 \\
\hline & $4 \mathrm{~g}$ & 2 & 27.027 & 0 & 31.494 & 1.32715 \\
\hline \multirow[t]{7}{*}{$\mathrm{pH}$} & $5 \mathrm{~g}$ & 2 & 29.9233 & 0.72338 & 31.3832 & 0.70831 \\
\hline & $6 \mathrm{~g}$ & 2 & 33.8235 & 0 & 33.8235 & 0 \\
\hline & $7 \mathrm{~g}$ & 2 & 37.8571 & 1.01015 & 42.8571 & 0 \\
\hline & $8 \mathrm{~g}$ & 2 & 40 & 0 & 50.7143 & 1.01015 \\
\hline & $9 \mathrm{~g}$ & 2 & 42.029 & 0 & 55.0725 & 0 \\
\hline & $10 \mathrm{~g}$ & 2 & 44.9275 & 0 & 57.2464 & 1.02479 \\
\hline & Total & 20 & & & & \\
\hline
\end{tabular}

Table 1: Result of $\%$ coagulation versus coagulant dosage for $\mathrm{pH}$.

\begin{tabular}{|c|c|c|c|c|c|c|}
\hline \multirow[b]{2}{*}{ Parameter } & \multirow{2}{*}{$\begin{array}{l}\text { Dosage } \\
\text { (g) }\end{array}$} & \multirow[b]{2}{*}{$\mathbf{N}$} & \multicolumn{2}{|l|}{$\mathrm{Al}_{2}\left(\mathrm{SO}_{4}\right)_{3} \cdot 18 \mathrm{H}_{2} \mathrm{O}$} & \multicolumn{2}{|l|}{$\mathrm{FeSO}_{4} \cdot 7 \mathrm{H}_{2} \mathrm{O}$} \\
\hline & & & Mean \% efficiency & Std. Deviation & Mean \% efficiency & Std. Deviation \\
\hline & $1 \mathrm{~g}$ & 2 & 13.333 & 0 & 30 & 4.714 \\
\hline & $2 \mathrm{~g}$ & 2 & 26.667 & 0 & 40 & 0 \\
\hline & $3 \mathrm{~g}$ & 2 & 33.333 & 0 & 46.667 & 0 \\
\hline & $4 \mathrm{~g}$ & 2 & 40 & 0 & 56.667 & 4.714 \\
\hline \multirow[t]{7}{*}{ TSS (mg/L) } & $5 \mathrm{~g}$ & 2 & 46.667 & 0 & 63.333 & 4.714 \\
\hline & $6 \mathrm{~g}$ & 2 & 67.708 & 1.4731 & 70.833 & 5.8926 \\
\hline & $7 \mathrm{~g}$ & 2 & 80 & 0 & 76.667 & 4.714 \\
\hline & $8 \mathrm{~g}$ & 2 & 86.667 & 0 & 80 & 9.4281 \\
\hline & $9 \mathrm{~g}$ & 2 & 93.333 & 0 & 86.667 & 9.4281 \\
\hline & $10 \mathrm{~g}$ & 2 & 98.71 & 0 & 96.548 & 0 \\
\hline & Total & 20 & & & & \\
\hline
\end{tabular}

Table 2: Result of \% coagulation versus coagulant dosage for TSS.

\begin{tabular}{|c|c|c|c|c|c|c|}
\hline \multirow[b]{2}{*}{ Parameter } & \multirow{2}{*}{$\begin{array}{l}\text { Dosage } \\
\text { (g) }\end{array}$} & \multirow[b]{2}{*}{$\mathbf{N}$} & \multicolumn{2}{|l|}{$\mathrm{Al}_{2}\left(\mathrm{SO}_{4}\right)_{3} \cdot 18 \mathrm{H}_{2} \mathrm{O}$} & \multicolumn{2}{|l|}{$\mathrm{FeSO}_{4} \cdot 7 \mathrm{H}_{2} \mathrm{O}$} \\
\hline & & & Mean \% efficiency & Std. Deviation & Mean \% efficiency & Std. Deviation \\
\hline & $1 \mathrm{~g}$ & 2 & 68.911 & 0 & 82.611 & 0 \\
\hline & $2 \mathrm{~g}$ & 2 & 71.429 & 0 & 85.07 & 0 \\
\hline & $3 \mathrm{~g}$ & 2 & 72.658 & 0 & 86.358 & 0 \\
\hline \multirow{2}{*}{$\mathrm{DO}$ (mg/L) } & $4 \mathrm{~g}$ & 2 & 76.405 & 0 & 88.817 & 0 \\
\hline & $5 \mathrm{~g}$ & 2 & 78.923 & 0 & 88.817 & 0 \\
\hline
\end{tabular}


Citation: Mbaeze MC, Agbazue VE, Orjioke NM (2017) Comparative Assessment of Performance of Aluminium Sulphate (Alum) and Ferrous Sulphate as Coagulants in Water Treatment. Mod Chem Appl 5: 233. doi: 10.4172/2329-6798.1000233

Page 5 of 14

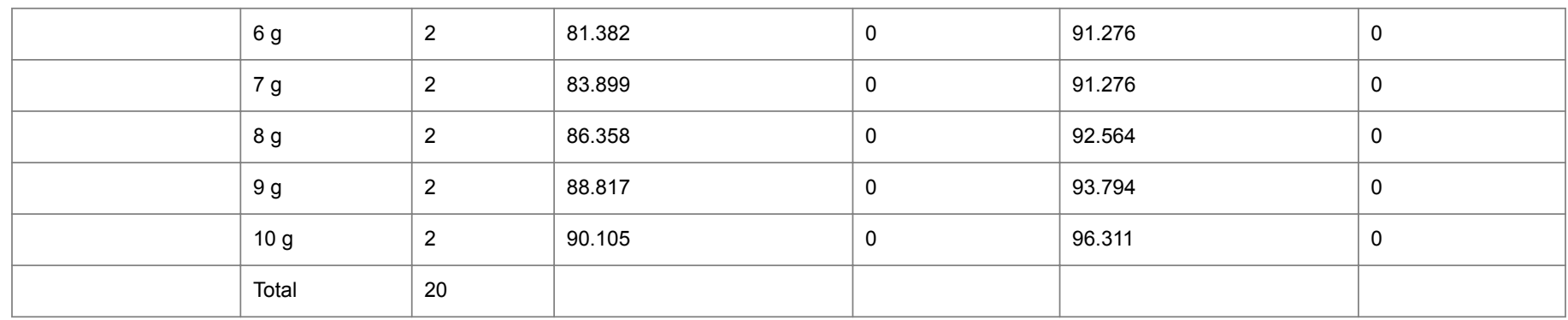

Table 3: Result of \% coagulation versus coagulant dosage for DO.

\begin{tabular}{|c|c|c|c|c|c|c|}
\hline \multirow[b]{2}{*}{ Parameter } & \multirow{2}{*}{$\begin{array}{l}\text { Dosage } \\
\text { (g) }\end{array}$} & \multirow[b]{2}{*}{$\mathbf{N}$} & \multicolumn{2}{|l|}{$\mathrm{Al}_{2}\left(\mathrm{SO}_{4}\right)_{3} \cdot 18 \mathrm{H}_{2} \mathrm{O}$} & \multicolumn{2}{|l|}{$\mathrm{FeSO}_{4} \cdot 7 \mathrm{H}_{2} \mathrm{O}$} \\
\hline & & & Mean \% efficiency & Std. Deviation & Mean $\%$ efficiency & Std. Deviation \\
\hline & $1 \mathrm{~g}$ & 2 & 79.717 & 0 & 84.9057 & 0 \\
\hline & $2 \mathrm{~g}$ & 2 & 84.9057 & 0 & 89.8113 & 0 \\
\hline & $3 \mathrm{~g}$ & 2 & 84.9057 & 0 & 95 & 0 \\
\hline & $4 \mathrm{~g}$ & 2 & 84.9057 & 0 & 97.3585 & 0 \\
\hline \multirow[t]{7}{*}{ BOD5 (mg/L) } & $5 \mathrm{~g}$ & 2 & 84.9057 & 0 & 97.3585 & 0 \\
\hline & $6 \mathrm{~g}$ & 2 & 84.9057 & 0 & 97.3585 & 0 \\
\hline & $7 \mathrm{~g}$ & 2 & 90.0943 & 0 & 97.3585 & 0 \\
\hline & $8 \mathrm{~g}$ & 2 & 95 & 0 & 100 & 0 \\
\hline & $9 \mathrm{~g}$ & 2 & 100 & 0 & 100 & 0 \\
\hline & $10 \mathrm{~g}$ & 2 & 100 & 0 & 100 & 0 \\
\hline & Total & 20 & & & & \\
\hline
\end{tabular}

Table 4: Result of $\%$ coagulation versus coagulant dosage for BOD5.

\begin{tabular}{|c|c|c|c|c|c|c|}
\hline \multirow[b]{2}{*}{ Parameter } & \multirow{2}{*}{$\begin{array}{l}\text { Dosage } \\
\text { (g) }\end{array}$} & \multirow[b]{2}{*}{$\mathbf{N}$} & \multicolumn{2}{|l|}{$\mathrm{Al}_{2}\left(\mathrm{SO}_{4}\right)_{3} \cdot 18 \mathrm{H}_{2} \mathrm{O}$} & \multicolumn{2}{|l|}{$\mathrm{FeSO}_{4} .7 \mathrm{H}_{2} \mathrm{O}$} \\
\hline & & & Mean $\%$ efficiency & Std. Deviation & Mean $\%$ efficiency & Std. Deviation \\
\hline & $1 \mathrm{~g}$ & 2 & 13.3333 & 0 & 30 & 4.71405 \\
\hline & $2 \mathrm{~g}$ & 2 & 26.6667 & 0 & 40 & 0 \\
\hline & $3 \mathrm{~g}$ & 2 & 33.3333 & 0 & 50.6667 & 0 \\
\hline & $4 \mathrm{~g}$ & 2 & 40 & 0 & 56.6667 & 4.71405 \\
\hline & $5 \mathrm{~g}$ & 2 & 46.6667 & 0 & 63.3333 & 4.71405 \\
\hline \multirow[t]{5}{*}{ Turbidity } & $6 \mathrm{~g}$ & 2 & 67.7083 & 1.47314 & 70.8333 & 5.89256 \\
\hline & $7 \mathrm{~g}$ & 2 & 80.6452 & 0 & 77.4194 & 4.56198 \\
\hline & $8 \mathrm{~g}$ & 2 & 86.6667 & 0 & 80 & 9.42809 \\
\hline & $9 \mathrm{~g}$ & 2 & 93.3333 & 0 & 86.6667 & 9.42809 \\
\hline & $10 \mathrm{~g}$ & 2 & 98.7097 & 0 & 96.7742 & 4.56198 \\
\hline
\end{tabular}


Citation: Mbaeze MC, Agbazue VE, Orjioke NM (2017) Comparative Assessment of Performance of Aluminium Sulphate (Alum) and Ferrous Sulphate as Coagulants in Water Treatment. Mod Chem Appl 5: 233. doi: 10.4172/2329-6798.1000233

\begin{tabular}{|l|l|l|l|l|l|l|}
\hline & Total & 20 & & & & \\
\hline
\end{tabular}

Table 5: Result of \% coagulation versus coagulant dosage for Turbidity.

\begin{tabular}{|c|c|c|c|c|c|c|}
\hline \multirow[b]{2}{*}{ Parameter } & \multirow{2}{*}{$\begin{array}{l}\text { Dosage } \\
\text { (g) }\end{array}$} & \multirow[b]{2}{*}{$\mathbf{N}$} & \multicolumn{2}{|l|}{$\mathrm{Al}_{2}\left(\mathrm{SO}_{4}\right)_{3} \cdot 18 \mathrm{H}_{2} \mathrm{O}$} & \multicolumn{2}{|l|}{$\mathrm{FeSO}_{4} \cdot 7 \mathrm{H}_{2} \mathrm{O}$} \\
\hline & & & Mean \% efficiency & Std. Deviation & Mean \% efficiency & Std. Deviation \\
\hline & $1 \mathrm{~g}$ & 2 & 57.627 & 0 & 60.8959 & 0 \\
\hline & $2 \mathrm{~g}$ & 2 & 69.73 & 0 & 66.707 & 0 \\
\hline & $3 \mathrm{~g}$ & 2 & 90 & 0 & 75.7869 & 0 \\
\hline & $4 \mathrm{~g}$ & 2 & 100 & 0 & 81.8402 & 0 \\
\hline \multirow[t]{7}{*}{ Chloride (mg/L) } & $5 \mathrm{~g}$ & 2 & 100 & 0 & 84.8668 & 0 \\
\hline & $6 \mathrm{~g}$ & 2 & 100 & 0 & 93.9467 & 0 \\
\hline & $7 \mathrm{~g}$ & 2 & 100 & 0 & 100 & 0 \\
\hline & $8 \mathrm{~g}$ & 2 & 100 & 0 & 100 & 0 \\
\hline & $9 \mathrm{~g}$ & 2 & 100 & 0 & 100 & 0 \\
\hline & $10 \mathrm{~g}$ & 2 & 100 & 0 & 100 & 0 \\
\hline & Total & 20 & & & & \\
\hline
\end{tabular}

Table 6: Result of $\%$ coagulation versus coagulant dosage for Chloride.

\begin{tabular}{|c|c|c|c|c|c|c|}
\hline \multirow[b]{2}{*}{ Parameter } & \multirow{2}{*}{$\begin{array}{l}\text { Dosage } \\
\text { (g) }\end{array}$} & \multirow[b]{2}{*}{$\mathbf{N}$} & \multicolumn{2}{|l|}{$\mathrm{Al}_{2}\left(\mathrm{SO}_{4}\right)_{3} \cdot 18 \mathrm{H}_{2} \mathrm{O}$} & \multicolumn{2}{|l|}{$\mathrm{FeSO}_{4} \cdot 7 \mathrm{H}_{2} \mathrm{O}$} \\
\hline & & & Mean \% efficiency & Std. Deviation & Mean \% efficiency & Std. Deviation \\
\hline & $1 \mathrm{~g}$ & 2 & 25 & 0 & 100 & 0 \\
\hline & $2 \mathrm{~g}$ & 2 & 50 & 0 & 100 & 0 \\
\hline & $3 \mathrm{~g}$ & 2 & 50 & 0 & 100 & 0 \\
\hline \multirow[t]{8}{*}{ Fluoride (mg/L) } & $4 \mathrm{~g}$ & 2 & 50 & 0 & 100 & 0 \\
\hline & $5 \mathrm{~g}$ & 2 & 100 & 0 & 100 & 0 \\
\hline & $6 \mathrm{~g}$ & 2 & 100 & 0 & 100 & 0 \\
\hline & $7 \mathrm{~g}$ & 2 & 100 & 0 & 100 & 0 \\
\hline & $8 \mathrm{~g}$ & 2 & 100 & 0 & 100 & 0 \\
\hline & $9 \mathrm{~g}$ & 2 & 100 & 0 & 100 & 0 \\
\hline & $10 \mathrm{~g}$ & 2 & 100 & 0 & 100 & 0 \\
\hline & Total & 20 & & & & \\
\hline
\end{tabular}

Table 7: Result of \% coagulation versus coagulant dosage for Fluoride.

\begin{tabular}{|l|l|l|l|l|l|l|}
\hline & Dosage & \multicolumn{3}{|l|}{} & $\mathrm{Al}_{2}\left(\mathbf{S O}_{4}\right)_{3} \cdot \mathbf{1 8 H}_{2} \mathbf{O}$ & \multicolumn{2}{l|}{$\mathrm{FeSO}_{4} \cdot \mathbf{7 \mathrm { H } _ { 2 } \mathbf { O }}$} \\
\hline Parameter & $\mathbf{( g )}$ & $\mathbf{N}$ & Mean \% efficiency & Std. Deviation & Mean \% efficiency & Std. Deviation \\
\hline & $1 \mathrm{~g}$ & 2 & 20 & 0 & 40 & 0 \\
\hline & $2 \mathrm{~g}$ & 2 & 28.8889 & 0 & 48.8889 & 0 \\
\hline
\end{tabular}


Citation: Mbaeze MC, Agbazue VE, Orjioke NM (2017) Comparative Assessment of Performance of Aluminium Sulphate (Alum) and Ferrous Sulphate as Coagulants in Water Treatment. Mod Chem Appl 5: 233. doi: 10.4172/2329-6798.1000233

Page 7 of 14

\begin{tabular}{|c|c|c|c|c|c|c|}
\hline & $3 \mathrm{~g}$ & 2 & 40 & 0 & 60 & 0 \\
\hline & $4 \mathrm{~g}$ & 2 & 48.8889 & 0 & 67.7778 & 0 \\
\hline \multirow[t]{7}{*}{ Phosphate (mg/L) } & $5 \mathrm{~g}$ & 2 & 71.1111 & 0 & 71.1111 & 0 \\
\hline & $6 \mathrm{~g}$ & 2 & 77.7778 & 0 & 75.5556 & 0 \\
\hline & $7 \mathrm{~g}$ & 2 & 77.7778 & 0 & 80 & 0 \\
\hline & $8 \mathrm{~g}$ & 2 & 80 & 0 & 88.8889 & 0 \\
\hline & $9 \mathrm{~g}$ & 2 & 80 & 0 & 91.1111 & 0 \\
\hline & $10 \mathrm{~g}$ & 2 & 80 & 0 & 91.1111 & 0 \\
\hline & Total & 20 & & & & \\
\hline
\end{tabular}

Table 8: Result of \% coagulation versus coagulant dosage for Phosphate.

\begin{tabular}{|c|c|c|c|c|c|c|}
\hline \multirow[b]{2}{*}{ Parameter } & \multirow{2}{*}{$\begin{array}{l}\text { Dosage } \\
\text { (g) }\end{array}$} & \multirow[b]{2}{*}{$\mathbf{N}$} & \multicolumn{2}{|l|}{$\mathrm{Al}_{2}\left(\mathrm{SO}_{4}\right)_{3} \cdot 18 \mathrm{H}_{2} \mathrm{O}$} & \multicolumn{2}{|l|}{$\mathrm{FeSO}_{4} \cdot 7 \mathrm{H}_{2} \mathrm{O}$} \\
\hline & & & Mean \% efficiency & Std. Deviation & Mean \% efficiency & Std. Deviation \\
\hline & $1 \mathrm{~g}$ & 2 & 19.986 & 0.0958 & 57.52 & 3.5451 \\
\hline & $2 \mathrm{~g}$ & 2 & 30.081 & 0.1916 & 67.412 & 3.5451 \\
\hline & $3 \mathrm{~g}$ & 2 & 39.973 & 0 & 79.946 & 0 \\
\hline \multirow[t]{8}{*}{$\mathrm{COD}(\mathrm{mg} / \mathrm{L})$} & $4 \mathrm{~g}$ & 2 & 50.136 & 0.1916 & 100 & 0 \\
\hline & $5 \mathrm{~g}$ & 2 & 69.851 & 0.0958 & 1000 & 0 \\
\hline & $6 \mathrm{~g}$ & 2 & 100 & 0 & 100 & 0 \\
\hline & $7 \mathrm{~g}$ & 2 & 100 & 0 & 100 & 0 \\
\hline & $8 \mathrm{~g}$ & 2 & 100 & 0 & 100 & 0 \\
\hline & $9 \mathrm{~g}$ & 2 & 100 & 0 & 100 & 0 \\
\hline & $10 \mathrm{~g}$ & 2 & 100 & 0 & 100 & 0 \\
\hline & Total & 20 & & & & \\
\hline
\end{tabular}

Table 9: Result of \% coagulation versus coagulant dosage for COD.

\begin{tabular}{|c|c|c|c|c|c|c|}
\hline \multirow[b]{2}{*}{ Parameter } & \multirow{2}{*}{$\begin{array}{l}\text { Dosage } \\
\text { (g) }\end{array}$} & \multirow[b]{2}{*}{$\mathbf{N}$} & \multicolumn{2}{|l|}{$\mathrm{Al}_{2}\left(\mathrm{SO}_{4}\right)_{3} \cdot 18 \mathrm{H}_{2} \mathrm{O}$} & \multicolumn{2}{|l|}{$\mathrm{FeSO}_{4} \cdot 7 \mathrm{H}_{2} \mathrm{O}$} \\
\hline & & & Mean \% efficiency & Std. Deviation & Mean \% efficiency & Std. Deviation \\
\hline & $1 \mathrm{~g}$ & 2 & 0 & 0 & 0 & 0 \\
\hline & $2 \mathrm{~g}$ & 2 & 0 & 0 & 0 & 0 \\
\hline & $3 \mathrm{~g}$ & 2 & 0 & 0 & 0 & 0 \\
\hline \multirow[t]{6}{*}{ Copper (mg/L) } & $4 \mathrm{~g}$ & 2 & 0 & 0 & 0 & 0 \\
\hline & $5 \mathrm{~g}$ & 2 & 0 & 0 & 0 & 0 \\
\hline & $6 \mathrm{~g}$ & 2 & 0 & 0 & 0 & 0 \\
\hline & $7 \mathrm{~g}$ & 2 & 0 & 0 & 0 & 0 \\
\hline & $8 \mathrm{~g}$ & 2 & 0 & 0 & 0 & 0 \\
\hline & $9 \mathrm{~g}$ & 2 & 0 & 0 & 0 & 0 \\
\hline
\end{tabular}


Citation: Mbaeze MC, Agbazue VE, Orjioke NM (2017) Comparative Assessment of Performance of Aluminium Sulphate (Alum) and Ferrous Sulphate as Coagulants in Water Treatment. Mod Chem Appl 5: 233. doi: 10.4172/2329-6798.1000233

Page 8 of 14

\begin{tabular}{|l|l|l|l|l|l|l|}
\hline & $10 \mathrm{~g}$ & 2 & 0 & 0 & 0 & 0 \\
\hline & Total & 20 & & & & \\
\hline
\end{tabular}

Table 10: Result of $\%$ coagulation versus coagulant dosage for Copper.

- Differences between the means were considered significant for P • Non-significant differences between the means were considered for values $<0.05$ $P$ values $>0.05$

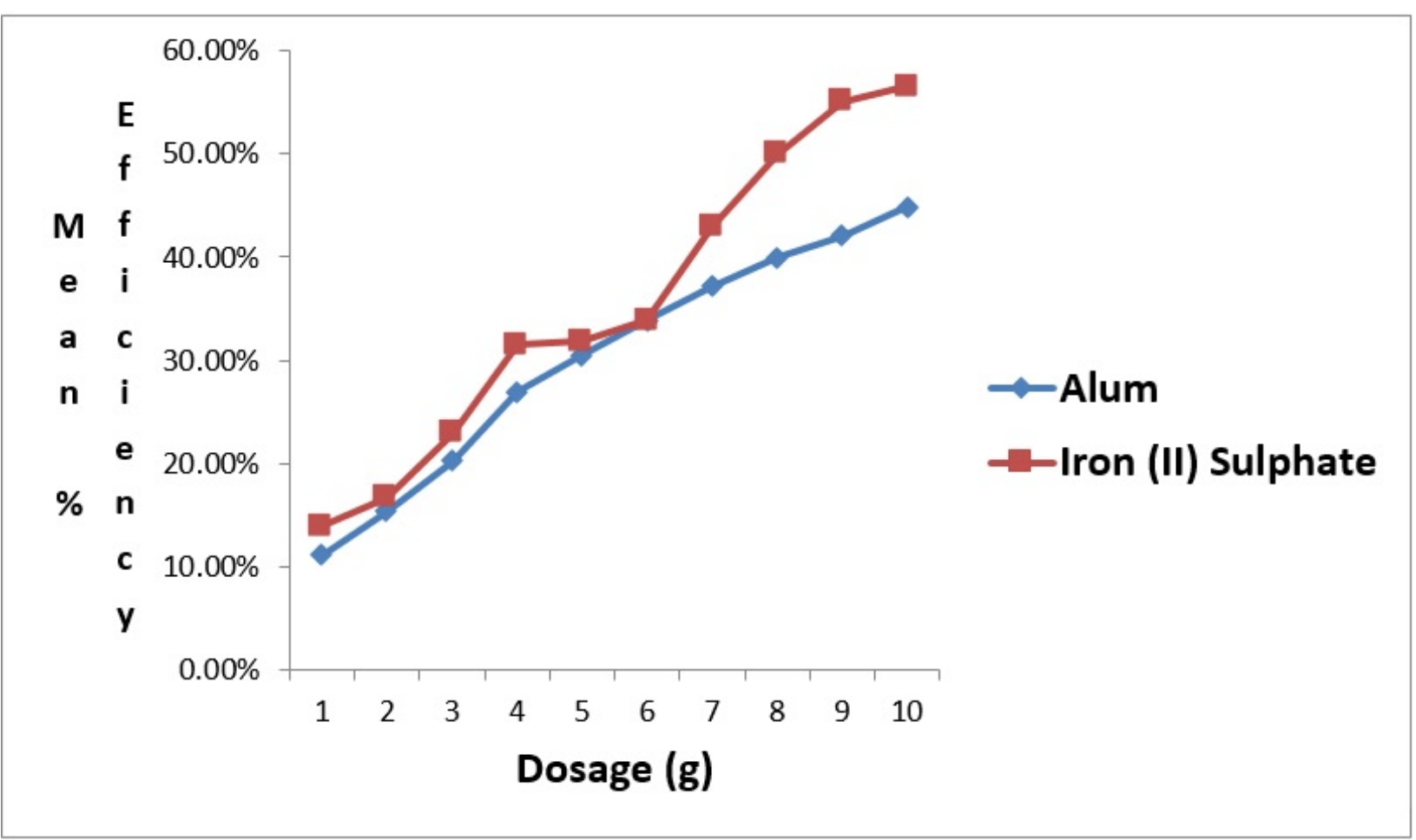

Figure 2: Comparative Variations of Mean \% Efficiency of $\mathrm{pH}$ with Dosage. 
Citation: Mbaeze MC, Agbazue VE, Orjioke NM (2017) Comparative Assessment of Performance of Aluminium Sulphate (Alum) and Ferrous Sulphate as Coagulants in Water Treatment. Mod Chem Appl 5: 233. doi: 10.4172/2329-6798.1000233

Page 9 of 14

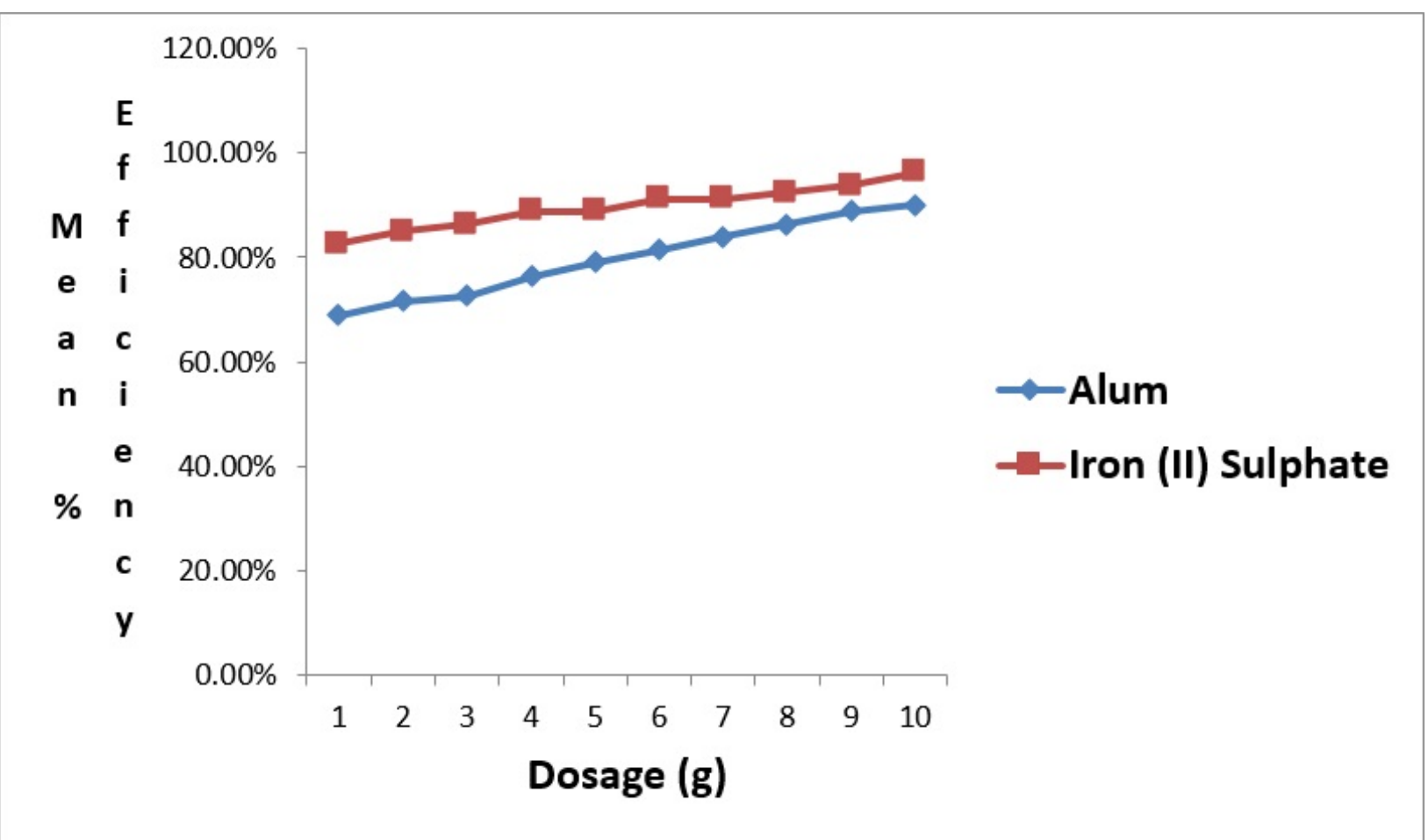

Figure 3: Comparative Variations of Mean \% Efficiency of TSS with Dosage.

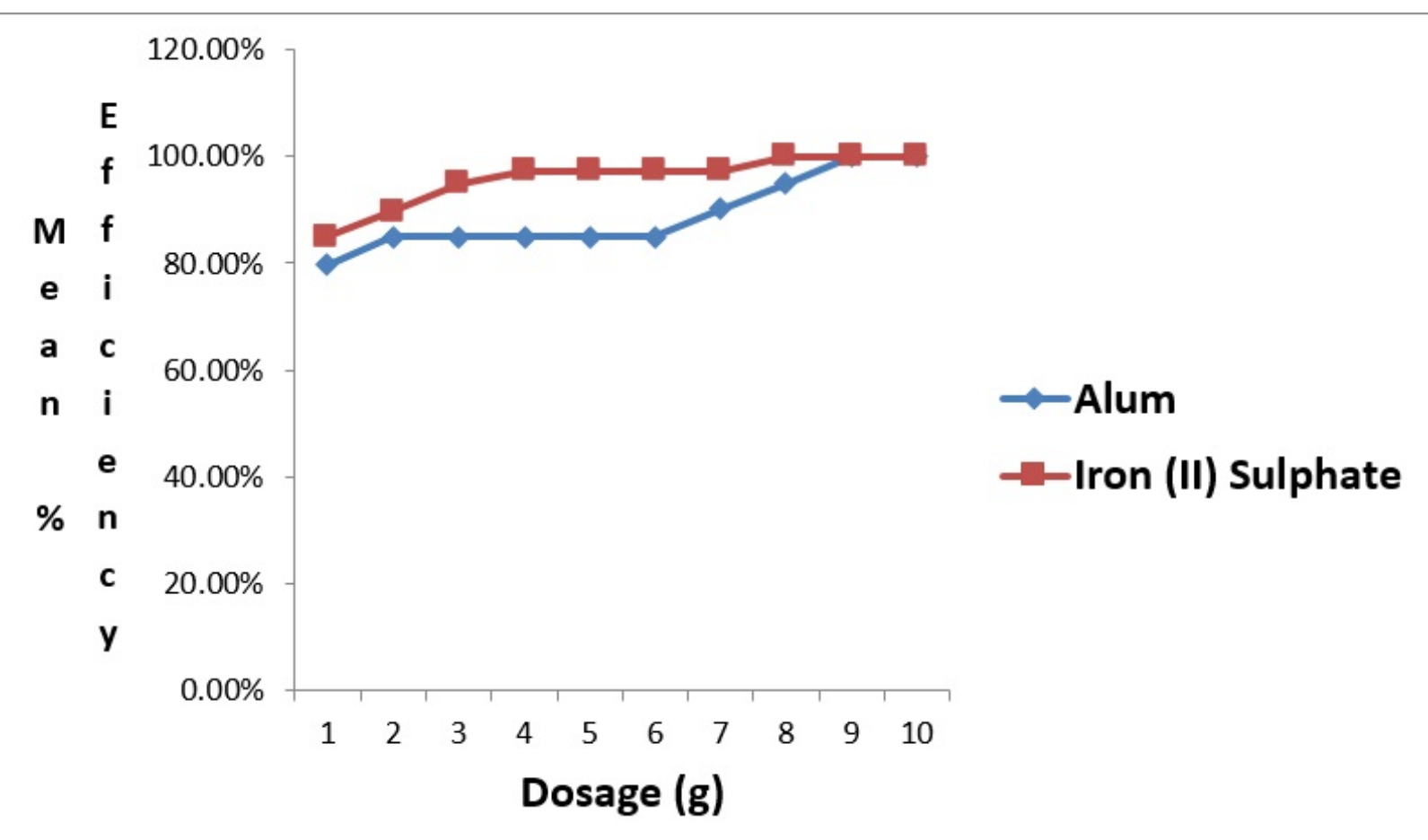

Figure 4: Comparative Variations of Mean \% Efficiency of DO with Dosage. 
Citation: Mbaeze MC, Agbazue VE, Orjioke NM (2017) Comparative Assessment of Performance of Aluminium Sulphate (Alum) and Ferrous Sulphate as Coagulants in Water Treatment. Mod Chem Appl 5: 233. doi: 10.4172/2329-6798.1000233

Page 10 of 14

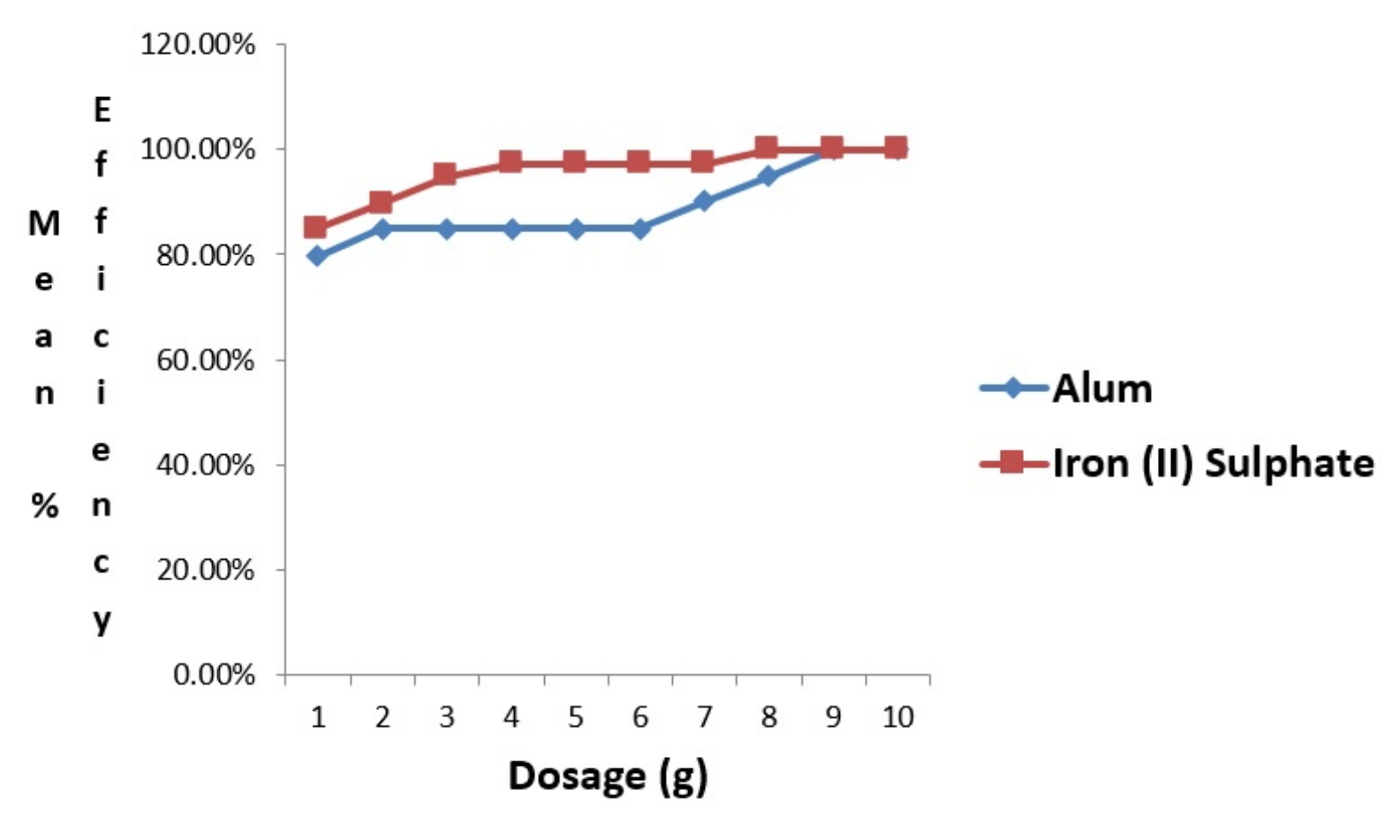

Figure 5: Comparative Variations of Mean \% Efficiency of BOD5 with Dosage.

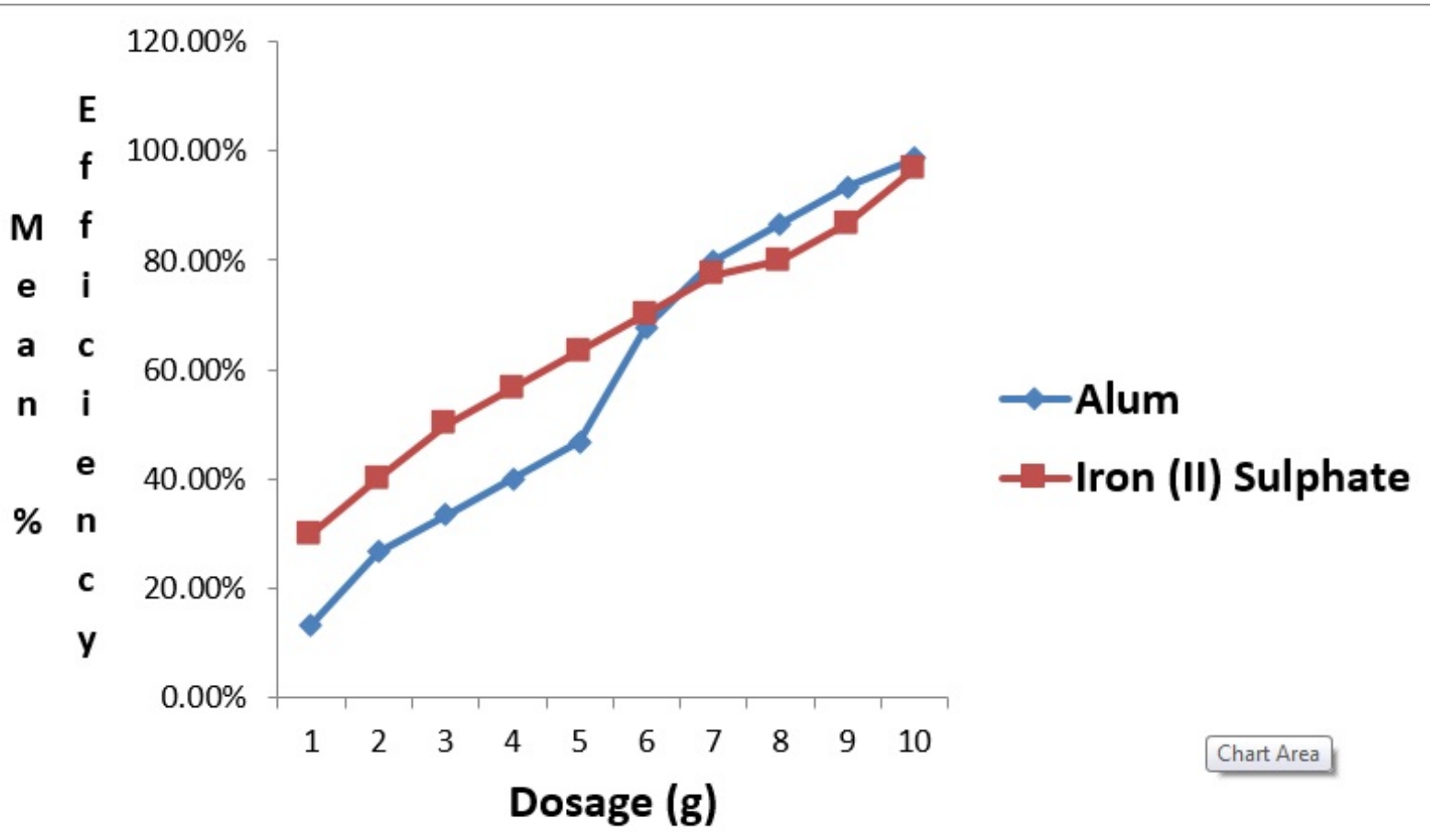

Figure 6: Comparative Variations of Mean \% Efficiency of Turbidity with Dosage. 
Citation: Mbaeze MC, Agbazue VE, Orjioke NM (2017) Comparative Assessment of Performance of Aluminium Sulphate (Alum) and Ferrous

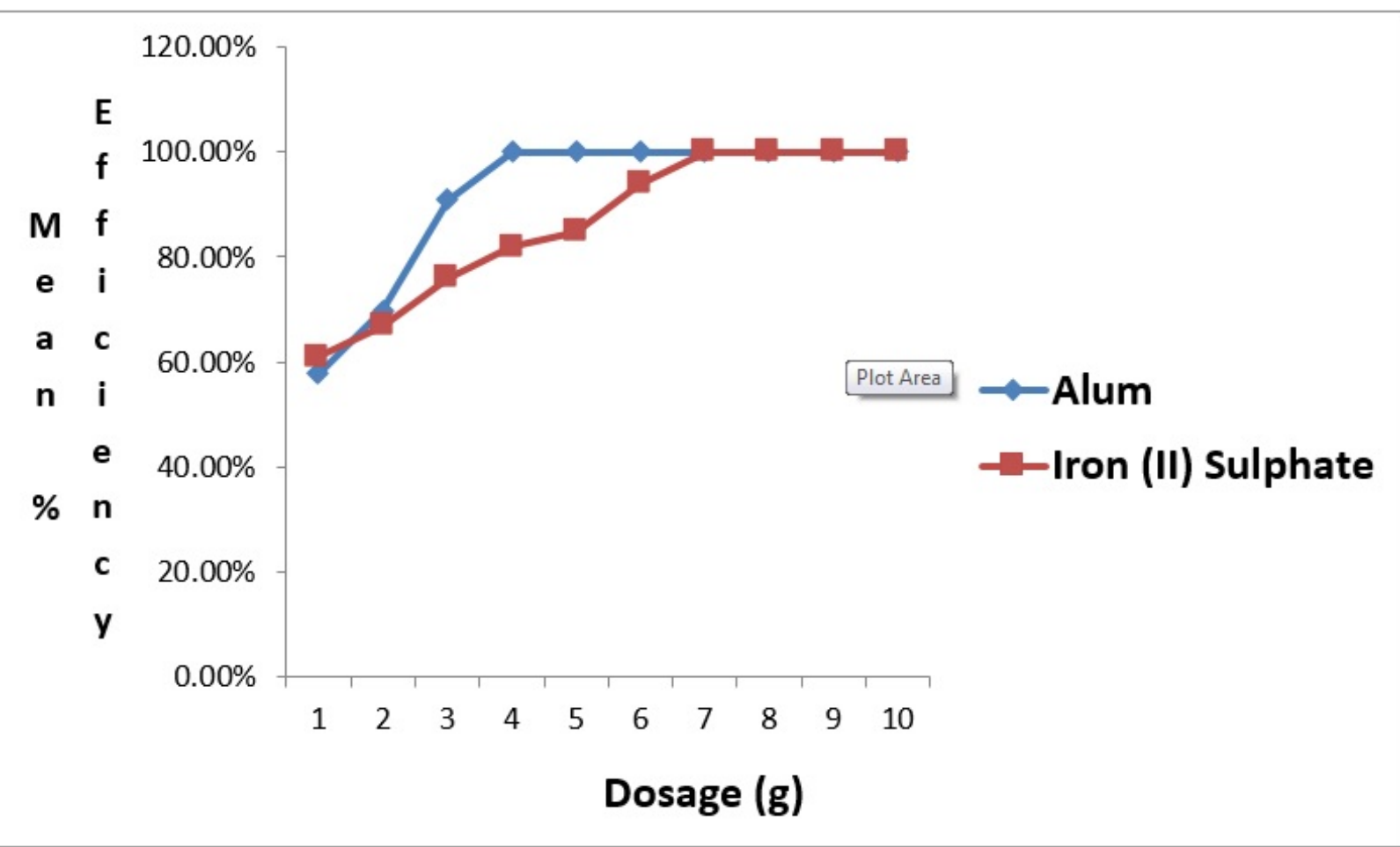

Figure 7: Comparative Variations of Mean \% Efficiency of Chloride with dosage.

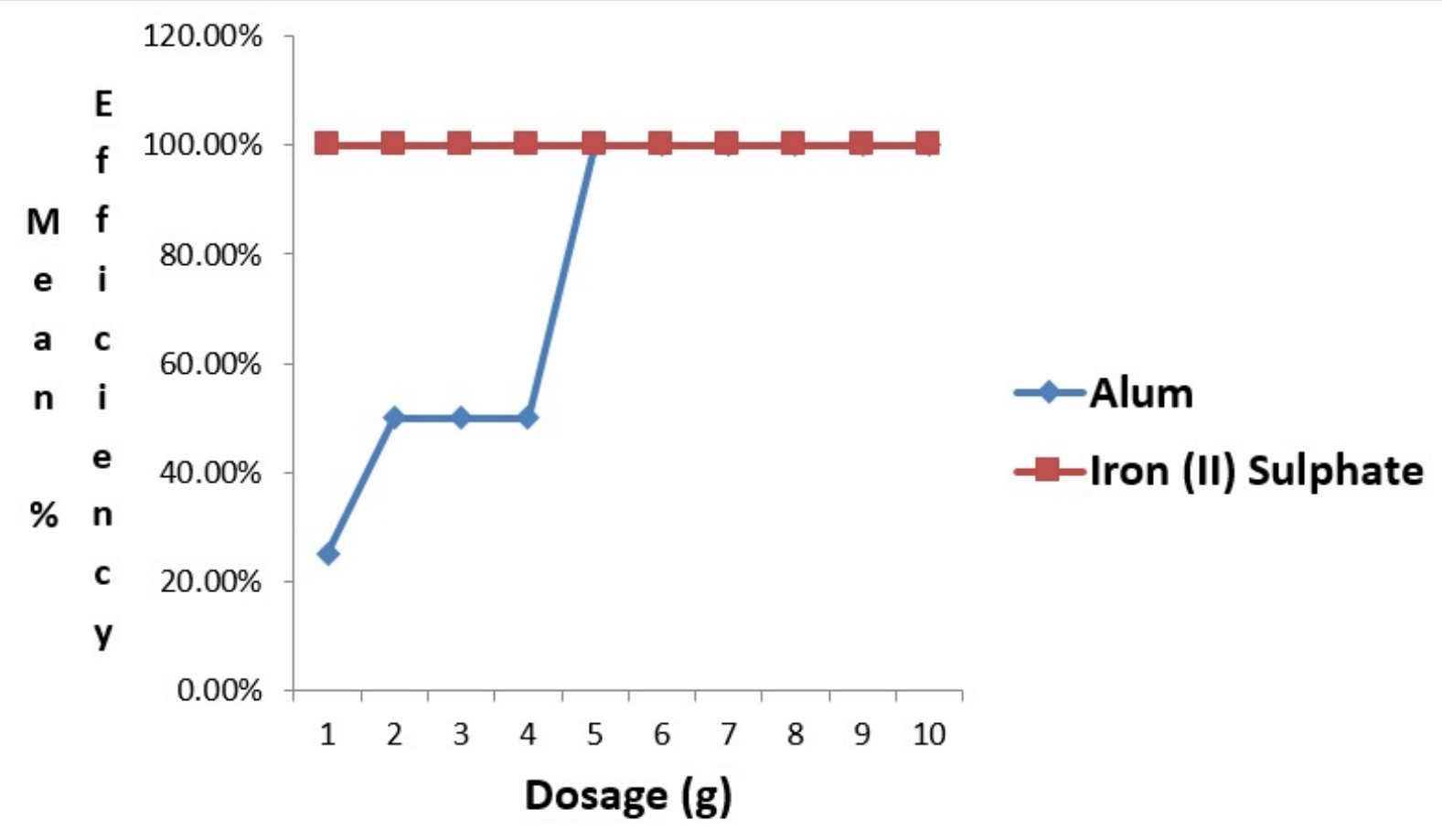

Figure 8: Comparative Variations of Mean \% Efficiency of Fluoride with Dosage. 
Citation: Mbaeze MC, Agbazue VE, Orjioke NM (2017) Comparative Assessment of Performance of Aluminium Sulphate (Alum) and Ferrous

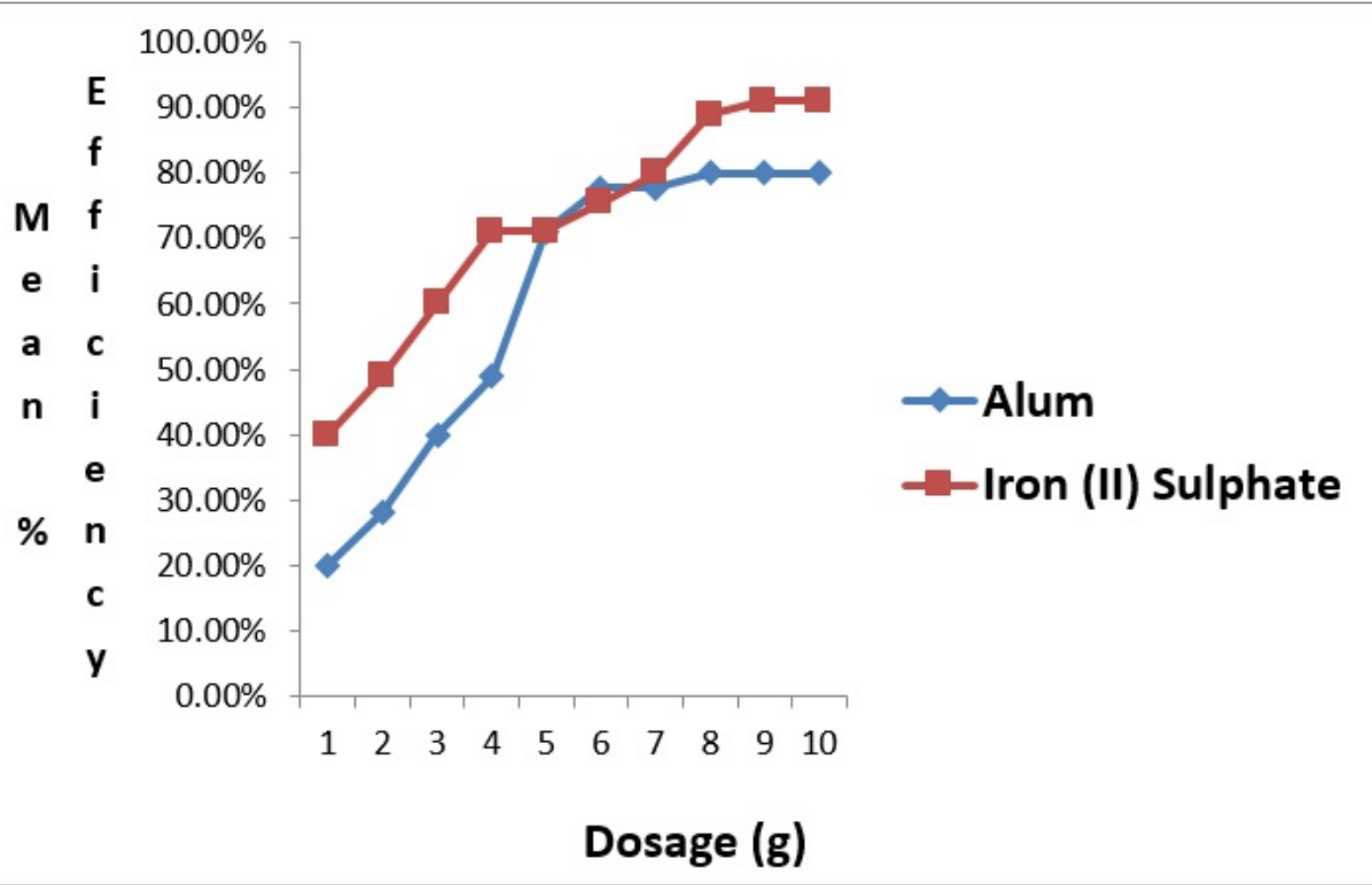

Figure 9: Comparative Variations of Mean \% Efficiency of Phosphate with Dosage.

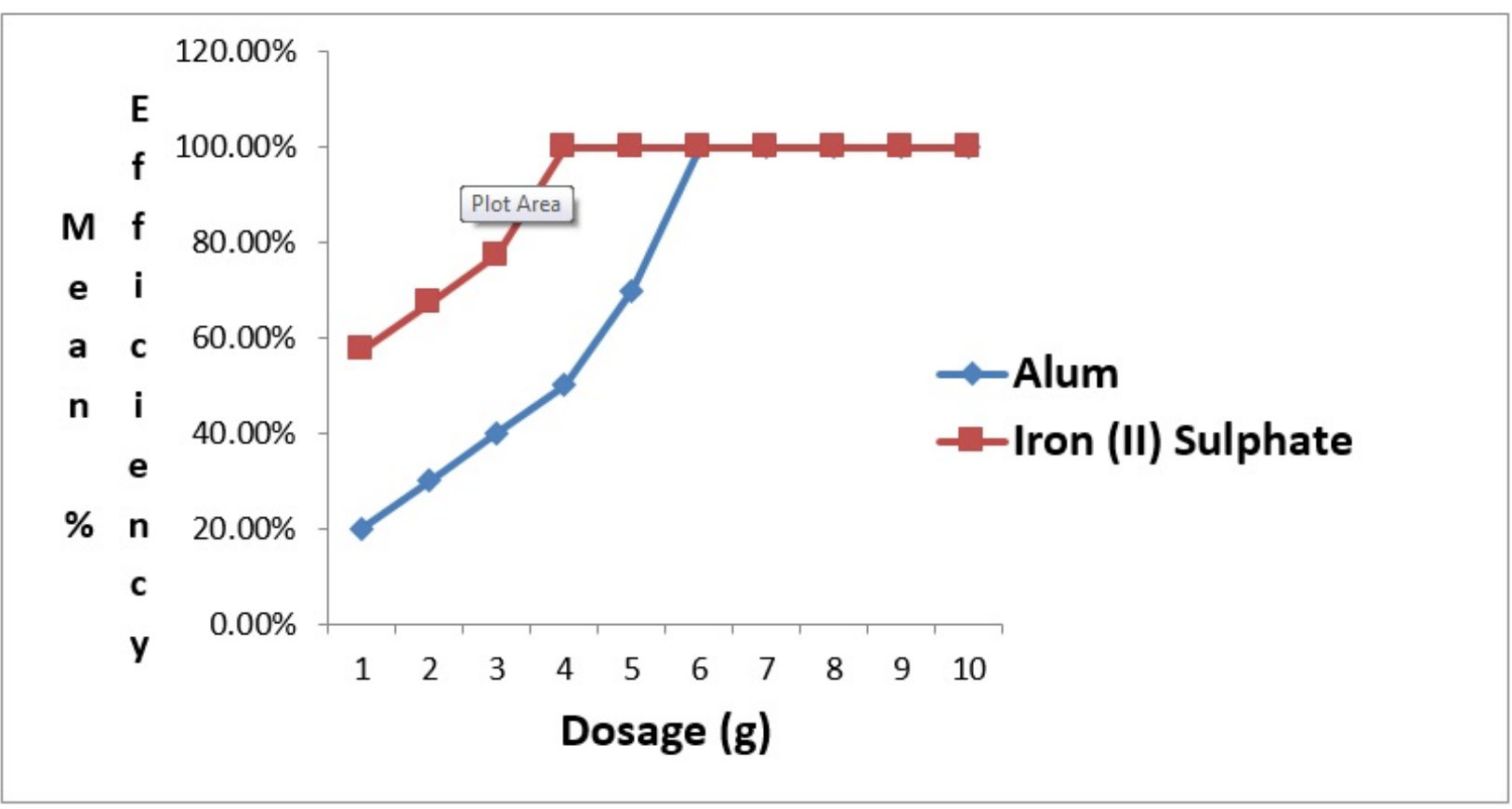

Figure 10: Comparative Variations of Mean \% Efficiency of COD with Dosage. 


\section{Discussion}

The preliminary assessed parameters of $\mathrm{pH}$, TSS, DO, BOD5, Turbidity, chloride, fluoride, phosphate, COD, showed high levels for each assessment, characteristic of turbid water. Post treatment analysis of turbid water showed that there were significant reductions in the parameters. This condition was more effective as the dosage of the coagulants (Alum and Iron (II) sulphate) increased from $1 \mathrm{~g}$ to $10 \mathrm{~g}$.

Chemical coagulant in turbid water treatment destabilizes the suspended contaminants such that the particles make contact and agglomerate, forming flocs that drop out of the solution by sedimentation. Highly charged hydrolyzed metal ions such as aluminium sulphate (alum) and partially hydrolyzed iron (II) sulphate in solution reduce the repulsive forces between colloids by compressing the diffusing double layer surrounding individual particles, and the forces of attraction cause these particles to shift to each other, producing progressive agglomeration. This was observed in turbid water treated with alum and iron (II) sulphate. The alum treated turbid water recorded a $\mathrm{pH}$ change from 7.4 in untreated sample to 3.8 in the treated at dosage of $1 \mathrm{~g}$ to $10 \mathrm{~g}$. This was corresponded to mean \% reduction of $\mathrm{pH}$ from $11.11 \%, 15.27 \%, 20.27 \%, 27.02 \%, 30.43 \%$, $33.82 \%, 37.14 \%, 40 \%, 42.02 \%$ to $44.93 \%$, while iron (II) sulphate treated turbid water recorded $\mathrm{pH}$ change from 7.4 in untreated sample to 3.0 in the treated at dosage of $1 \mathrm{~g}$ to $10 \mathrm{~g}$, which corresponded to mean \% reduction of $\mathrm{pH}$ from $13.88 \%, 16.66 \%, 22.97 \%, 31.50 \%$, $31.88 \%, 33.82 \%, 42.85 \%, 50 \%, 55.07 \%$ to $56.52 \%$. $\mathrm{pH}$ is a measure of acidity or alkalinity of a substance in solution.

It was observed that the $\mathrm{pH}$ level using iron (II) sulphate coagulant differed significantly $(\mathrm{p}<0.05)$ among dosages $1 \mathrm{~g}$ to $5 \mathrm{~g}$ when compared with $\mathrm{pH}$ level using alum of the same dosage $1 \mathrm{~g}$ to $5 \mathrm{~g}$. This explained why increased dosages caused mean \% reduction in $\mathrm{pH}$ and variability of heavy metals. At dosage of $6 \mathrm{~g}$, there was no significant $(\mathrm{p}>0.05)$ increase as both coagulants had the same mean \% reduction $33.8235 \%$. After $6 \mathrm{~g}$ dosage, there was a significant $(\mathrm{p}<0.05)$ improvement on $\mathrm{pH}$ level using iron (II) sulphate coagulant when compared with $\mathrm{pH}$ level using alum of the same dosage. Furthermore, the water appeared to be acidic in all dosages as the measured values of $\mathrm{pH}$ were below WHO guidelines. The findings suggested that the treated water is unsafe for drinking, and so required controlled $\mathrm{pH}$ adjustment for alum and iron (II) sulphate coagulation.

The total suspended solids of turbid water changed from 15500 $\mathrm{mg} / \mathrm{L}$ in untreated to $200 \mathrm{mg} / \mathrm{L}$ in the treated at dosage of $1 \mathrm{~g}$ to $10 \mathrm{~g}$ of alum, which represented mean $\%$ efficiency of $13.33 \%, 26.66 \%, 33.33 \%$, $40 \%, 46.66 \%, 67.74 \%, 80.64 \%, 86.66 \%, 93.33 \%$ and $98.70 \%$. Guida et al. (2005), indicated that alum effectively remove COD (65\%) and TSS $(>75 \%)$ on the average value of COD using $150 \mathrm{mg} / \mathrm{L}$ alum at a $\mathrm{pH}$ range of 5-8. The removal efficiency for $1-10 \mathrm{~g}$ of iron (II) sulphate changed from $15000 \mathrm{mg} / \mathrm{L}$ in untreated sample to $500 \mathrm{mg} / \mathrm{L}$ in treated samples which represented the mean \% efficiency of $30 \%, 40 \%, 50 \%$, $56.66 \%, 63.33 \%, 70 \%, 77.42 \%, 80 \%, 86.66 \%$ and $96.77 \%$. The TSS level of iron (II) sulphate showed a significant $(\mathrm{p}<0.05)$ increase among the dosages of $1 \mathrm{~g}$ to $6 \mathrm{~g}$ when compared with alum. At higher dosages of 7-10 g, alum showed significant $(\mathrm{p}<0.05)$ increases over iron (II) sulphate at same dosage of 7-10 g of iron (II) sulphate. It was observed that at higher dosage of iron (II) sulphate, turbidity occurred in the treated water, and thus caused increase in TSS. This explained the reason why alum is used mostly in water treatment plants for purification processes.
The dissolved oxygen is a measure of the amount of oxygen required by bacteria for oxidation of waste. The dissolved oxygen (DO) from the untreated sample was $17.08 \mathrm{mg} / \mathrm{L}$ to $1.69 \mathrm{mg} / \mathrm{L}$ in treated at dosage of 1 $\mathrm{g}$ to $10 \mathrm{~g}$ of alum representing mean \% efficiencies of $68.91 \%, 71.42 \%$, $72.65 \%, 76.40 \%, 78.92 \%, 81.38 \%, 83.89 \%, 86.35 \%, 88.81 \%$ and $90.10 \%$ while mean \% efficiency for the same dosages of iron (II) sulphate gave $82.61 \%, 85.07 \%, 86.35 \%, 88.81 \%, 88.81 \%, 91.27 \%, 91.27 \%, 92.56 \%$, 93.79\% and $96.31 \%$. However, the dissolved oxygen (DO) level of iron (II) sulphate differed significantly $(\mathrm{p}<0.05)$ in all dosages $1 \mathrm{~g}$ to $10 \mathrm{~g}$ when compared with alum of the same dosages 1 to 10 . The study revealed that iron (II) sulphate is more efficient in removal of dissolved oxygen than alum.

Similarly, the Biochemical Oxygen Demand (BOD5) was one of the parameters employed to measure the change over 5 days in dissolved $\mathrm{O}_{2}$ (DO) concentration in a stoppered bottle completely filled with the water sample. The value of BOD5 in untreated sample revealed 10.60 $\mathrm{mg} / \mathrm{L}$ to $0.00 \mathrm{mg} / \mathrm{L}$ in the treated samples at dosages of $1 \mathrm{~g}$ to $10 \mathrm{~g}$ of alum which represented mean \% efficiences of $79.71 \%, 84.90 \%$, $84.90 \%, 84.90 \%, 84.90 \%, 84.90 \%, 90.09 \%, 95 \%, 100 \%$ and $100 \%$. The mean \% efficiency of iron (II) sulphate at the same dosages showed $84.90 \%, 89.81 \%, 95 \%, 97.35 \%, 97.35 \%, 97.35 \%, 97.35 \%, 100 \%, 100 \%$ and $100 \%$. It was observed that at respective dosages of $2 \mathrm{~g}, 3 \mathrm{~g}, 4 \mathrm{~g}, 5 \mathrm{~g}$ and $6 \mathrm{~g}$ alum had the same mean \% efficiency of $84.90 \%$. This is because of the fact that sulphur-based chemicals are oxygen scavengers (Hill 2003). In a similar way, iron (II) sulphate had similar efficiency of $97.35 \%$ at respective dosages of $4 \mathrm{~g}, 5 \mathrm{~g}, 6 \mathrm{~g}$ and $7 \mathrm{~g}$. At a dosage of $9 \mathrm{~g}$, alum attained optimum efficiency for complete BOD5 removal, while iron (II) sulphate achieved optimum efficiency at dosage of $8 \mathrm{~g}$ for BOD5 extinction and the water freed from organic pollutants. At this point the tendency of the treated water to form Disinfection ByProducts is nill. Finally, the Biochemical Oxygen Demand (BOD5) of iron (II) sulphate showed a significant $(\mathrm{p}<0.05)$ increase in all dosages $1 \mathrm{~g}$ to $10 \mathrm{~g}$ when compared with alum of the same dosages.

In chloride removal, the chloride recorded $8.26 \mathrm{mg} / \mathrm{L}$ from untreated to $0.00 \mathrm{mg} / \mathrm{L}$ in the treated at dosages of $1 \mathrm{~g}$ to $10 \mathrm{~g}$ of alum. These corresponded to mean \% efficiency of $57.63 \%, 69.73 \%, 90.92 \%$, $100 \%, 100 \%, 100 \%, 100 \%, 100 \%, 100 \%$ and $100 \%$, while iron (II) sulphate revealed mean \% efficiency of $60.89 \%, 66.70 \%, 75.78 \%$, $81.84 \%, 84.86 \%, 93.95 \%, 100 \%, 100 \%, 100 \%$ and $100 \%$ for the same dosage range. It was observed that at dosage of $4 \mathrm{~g}$ alum achieved optimum efficiency while iron (II) sulphate achieved optimum efficiency (100\%) at dosage of $7 \mathrm{~g}$. The chloride level of iron (II) sulphate at $1 \mathrm{~g}$ dosage showed a significant $(\mathrm{p}<0.05)$ increase when compared with alum of the same dosage. At dosages of 2-10 g, the chloride level of alum differed significantly $(p<0.05)$ when compared with the chloride level of iron (II) sulphate. This study suggested that alum has more adsorptive capacity for chloride than iron (II) sulphate.

However, in 1975, the EPA named fluoride as a contaminant in the National Interim Primary Drinking Water Regulations. A maximum contaminant level (MCL) was set at $1.4-2.4 \mathrm{mg} / \mathrm{L}$ to prevent dental fluorosis and more serious effects. To balance the benefits of fluoride for dental health, the deleterious effect of ingesting too much fluoride, and the costs of removing high concentrations of naturally occurring fluoride, the EPA in 1985 issued a new MCL of $4 \mathrm{mg} / \mathrm{L}$ for fluoride with secondary MCL of $2 \mathrm{mg} / \mathrm{L}$. Systems with fluoride levels between 2 $\mathrm{mg} / \mathrm{L}$ and $4 \mathrm{mg} / \mathrm{L}$ must provide the public with information about possible tooth discoloration. The best available technologies for fluoride removal from water are generally considered to be activated alumina adsorption and reverse osmosis. 
In some cases, fluoride removal by aluminium and iron have been shown to be cost effective. It was recorded that fluoride in untreated sample was $2.00 \mathrm{mg} / \mathrm{L}$ to $0.00 \mathrm{mg} / \mathrm{L}$ in the treated at dosages of $1 \mathrm{~g}$ to $10 \mathrm{~g}$ of iron (II) sulphate representing mean \% efficiency of $100 \%$, $100 \%, 100 \%, 100 \%, 100 \%, 100 \%, 100 \%, 100 \%$ and $100 \%$, while alum of the same dosages achieved mean $\%$ efficiency of $25 \%, 50 \%, 50 \%, 50 \%$, $100 \%, 100 \%, 100 \%, 100 \%, 100 \%, 100 \%$. The fluoride level using iron (II) sulphate coagulant differed significantly $(\mathrm{p}<0.05)$ when compared with fluoride level of alum at $1 \mathrm{~g}$ to $4 \mathrm{~g}$ and there was no significant ( $\mathrm{p}>0.05$ ) increase as from $5 \mathrm{~g}$ to $10 \mathrm{~g}$ dosages. Therefore iron (II) sulphate coagulant was more efficient in fluoride removal compared to the aluminium sulphate coagulant.

The degree of phosphorus removal depends not only on the coagulant added, but also on the mode of solid-liquid separation employed (Yukselen and Gregory, 2004). It was recorded that phosphorous content in untreated sample was $9.00 \mathrm{mg} / \mathrm{L}$ to $0.80 \mathrm{mg} / \mathrm{L}$ in treated sample at dosage of 1-10 g of iron (II) sulphate which corresponded to mean $\%$ efficiency of $40 \%, 48.88 \%, 60 \%, 71.11 \%$, $71.11 \%, 75.55 \%, 80 \%, 88.88 \%, 91.11 \%$ and $91.11 \%$, while mean $\%$ efficiency of alum for the same dosages were $20 \%, 28 \%, 40 \%, 48.88 \%$, $71.11 \%, 77.77 \%, 80 \%, 80 \%$ and $80 \%$. The phosphate level using iron (II) sulphate coagulant showed a significant $(\mathrm{p}<0.05)$ increase in dosages $1 \mathrm{~g}$ to $4 \mathrm{~g}$ when compared with alum of the same dosages. At dosage $5 \mathrm{~g}$, there was no significant difference, and at $6 \mathrm{~g}$ dosage, alum showed significant improvement over iron (II) sulphate. At higher dosage of $7 \mathrm{~g}, 8 \mathrm{~g}, 9 \mathrm{~g}$ and $10 \mathrm{~g}$, the phosphate level using iron (II) sulphate coagulant showed significant $(p<0.05)$ increase when compared with phosphate level of alum. The study suggested that iron (II) sulphate is more efficient in phosphate removal compared to alum.

From the result of COD, it was observed that the COD level using iron (II) sulphate coagulant showed significant $(\mathrm{p}<0.05)$ increase among dosages of 1-3 g when compared with alum of the same dosages. At dosages of $4 \mathrm{~g}$ to $10 \mathrm{~g}$, iron (II) sulphate attained optimum efficiency of $100 \%$ while alum achieved optimum efficiency at $6 \mathrm{~g}$ to 10 g. Again, the optimum dose of coagulant enhanced maximum removal of COD.

Finally, copper values in the untreated sample were recorded 0.001 $\mathrm{mg} / \mathrm{L}$. The same values were obtained in the treated water sample of $1 \mathrm{~g}$ to $10 \mathrm{~g}$ of alum which implied that the copper was below the limit of detection. In the case of iron (II) sulphate coagulant, there was no difference between the untreated and treated samples. The obvious indication of these results is that copper was totally absent in both the humus soil and the base water sample.

\section{Conclusion and Recommendations}

Comparative Assessment of Performance of Aluminium Sulphate (Alum) and Ferrous Sulphate as Coagulants in Water Treatment was carried out on turbid water, using sedimentation beaker experiments. Among the range of water quality parameters studied, the results clearly indicated the comparative strengths and weaknesses of the two coagulants, in terms of coagulation (or removal) efficiency. The overall results of the coagulation studies showed that coagulation efficiency is parameter dependent. It is suggested that a combination of lime as a coagulant aid with any of these coagulants could improve efficiency in some parameters. Furthermore, it is proposed that application of alterative coagulants like moringa oleifera and prosopis affricana peel powder need to be properly evaluated at industrial scale, in order to increase the range of coagulant easily available for water treatment. This is to ensure the efficiency safety, efficacy and quality of treated water. Finally, it is recommended that the outcome of this work can be an important guide to water treatment operators.

\section{References}

1. Leiknes T, Odegaard H, Myklebust H (2004) Removal of Natural Organic Matter (NOM) in drinking water treatment by coagulationmicrofiltration using metal membranes. J Membr Sci 242: 47-55.

2. Matilainen A, Lindovist N, Tuhkanen T (2005) Comparison of the efficiency of Aluminium and Ferric sulphate in the Removal of Natural Organic Matter During Drinking Water Treatment Process. Environ Technol 26: 867-875.

3. Chowdhury S, Champagne P, Mclelland PJ (2009) Models for predicting disinfection by product (DBP) formation in drinking waters: A Chronological Review. Sci Total Environ 407: 4189-4206.

4. Hsu CH, Jeng WL, Chang RM, Chien LC, Han BC (2001) Estimation of potential lifetime cancer risk for THM from consuming chlorinated drinking water in Taiwan. J Environ Res 85: 77-82.

5. USEPA (2003) National Primary Drinking water regulations: stage 2 Disinfectants and Disinfection By-product Rule: proposed Rule. Federal Register: 49547-49681.

6. Wu G, Lo S (2010) Effects of data normalization and inherent-factor on decision of optimal coagulant dosage in water treatment by artificial neural network. Expert Syst Appl 37: 4974-4983.

7. Zainal-Abideen M, Aris A, Yusof F, Abdul-Majid A, Selamat A (2012) Optimizing the Coagulation process in a drinking water treatment plant comparison between traditional and statistical experiment as design jar test. Water Sci Technol 65: 3.

8. Joo DS, Choi DJ, Park H (2000) The effects of data processing in the determination coagulant dosing rate. Water Res 34: 3295-3302.

9. Yu RF, Kang SF, Liaw SL, Chen MC (2000) Application of Artificial neural network to control the coagulant dosing in water treatment plant. Water Sci Technol 42: 403-408.

10. Maier HR, Morgan N, Chow CWK (2004) Use of Artificial Neural networks for predicting optimal alum doses and treated water quality parameters. Environ Model Softw 19: 485-494.

11. Gholikandi GB, Delnavaz M, Riahi R (2011) Use of Artificial Neural Network for prediction of Coagulation/Flocculation process by Pac in water Treatment Plant. Environ Eng Manag J 10: 1719-1725.

12. Robenson AB (2008) Development of Neural Network Based Estimator to Determine Coagulant Dosage and treated water qualities in a water treatment plant.

13. Wu G, Lo S (2008) Predicting real-time coagulant dosage in water treatment by Artificial neural networks and adaptive network-based fuzzy inference system. Eng Appl Artif intell 21: 1189-1195.

14. Gebbie P (2006) An Operator's Guide to Water Treatment Coagulants, 31st Annual Water Industry Workshop-Operations Skills, University Central Queensland Campus-Rockhampton.

15. Effluent Treatment: Coagulation By: Sivarama Krishnan CN. 